\title{
Actividades de la vida diaria
}

\author{
Dulce María Romero Ayuso*
}

Universidad de Castilla-La Mancha

\begin{abstract}
Resumen: Las actividades de la vida diaria constituyen un tema de interés especialmente para los profesionales vinculados al ámbito de la rehabilitación. A pesar de su relevancia tanto social como académica son escasas las publicaciones específicas sobre este tópico. En el presente trabajo se revisan las aportaciones y visión de la actividad humana como objeto de estudio de la psicología, la taxonomía de las actividades de la vida diaria y su relación con las habilidades para la vida independiente. De acuerdo con las propuestas más recientes, el estudio de las actividades de la vida diaria incluye, por un lado, la consideración de tres elementos: las destrezas de una determinada persona, las tareas u operaciones a realizar y el contexto donde se lleva a cabo; y por otro lado, la interacción de los tres factores.

Palabras clave: Actividad; actividades de la vida diaria; habilidades adaptativas; independencia personal.
\end{abstract}

\section{Introducción}

A lo largo del siglo pasado, especialmente después de 1917 distintos profesionales afines al ámbito de las ciencias de la salud y del trabajo social comenzaron a considerar la importancia que tenía para las personas las diferentes actividades que realizaban cotidianamente y que por algún acontecimiento inesperado, ya desde la gestación o a lo largo del desarrollo, suponían un reto difícilmente salvable sin la ayuda profesional y del entorno social más próximo.

Los diferentes avances en el ámbito de la biomedicina, el aumento de la esperanza de vida y el estado del bienestar también han contribuido a que en el mundo occidental uno de los valores más preciados sea la independencia y autonomía personal. Los conceptos de independencia y autonomía personal inexorablemente están unidos al de actividades de la vida diaria. De hecho, la independencia y autonomía personal se refiere a la misma en las actividades de la vida diaria. Parece, por tanto, oportuno detenerse en conocer qué son las actividades de la vida diaria. A lo largo de este trabajo intentaremos hacer una primera aproximación al armazón teórico que sustenta el estudio de la actividad y, posteriormente, a las particularidades de las actividades de la vida diaria en la psicología contemporánea. Finalmente, revisaremos los encuentros y desencuentros entre el concepto de habilidad para la vida diaria y actividades de la vida diaria.

\section{El estudio de la actividad humana}

A pesar de que distintas perspectivas psicológicas han ido enfatizando con mayor o menor interés la relevancia de la acción y la actividad como tema del estudio psicológico. Son escasas las aportaciones que consideran a las actividades humanas globalmente y no divididas en procesos.

* Dirección para correspondencia [Correspondence address]: Dulce María Romero Ayuso. Universidad de Castilla-La Mancha. Avda. Real Fábrica de Sedas s/n. 45600 - Talavera de la Reina (Toledo, España). E-mail: Dulce.Romero@uclm.es
Title: Activities of daily living.

Abstract: The activities of daily living are an interesting topic specially for professional linking to the rehabilitation work. In spite of their social and academic relevance are few specific publications about this. In this manuscript we review the contributions and the perspective of human activity as an object of study from psychology, the taxonomy of activities of daily living and their relations with the abilities to independent live. According to the new approaches, the study of activities of daily living includes three elements: on one hand the individual capacities or abilities, the tasks and the context or environmental; and the other hand the interaction between the subject skills.

Key words: Activity; activities of daily living; adaptive skills; personal independent.

En primer lugar revisaremos la atención prestada por la psicología de la actividad a la funcionalidad humana. Seguidamente revisaremos el paradigma de la psicología cognitiva, adentrándonos en aquellos detalles que son esenciales para entender la actividad humana.

Canter (Canter, 1985) indica que una de las ventajas de centrarse sobre el término acción mejor que en el término conducta, es que se encuentra implicada una clara distinción entre el estudio psicológico convencional de respuestas y movimientos motores (lo que se denomina habitualmente conducta) y el estudio de las secuencias localizadas de actividad humana. Afirma que no es necesario ocuparse de las conductas limitadas de un organismo aislado, pudiendo en su lugar centrar la atención de la psicología legítimamente en las actividades y experiencias humanas dentro de sus marcos centrales. También es oportuno mencionar a la psicología cultural, que rescata la intencionalidad y subraya el papel de los significados en toda acción humana, acorde con la psicología soviética (Bruner, 1998).

$\mathrm{Y}$ aunque todavía hay una ausencia de una teoría global de la actividad humana que pueda ser considerada como un nuevo paradigma, han existido en el siglo recientemente pasado distintos intentos por abordarla (Mayor, 1985).

Dentro de estos intentos, cabe destacar a la psicología soviética que entiende que uno de los objetivos de la psicología recae en el estudio de la estructura de la actividad humana. Dentro de la psicología soviética, cabe destacar la teoría de la actividad de Leontiev (Leontiev, 1989) que fue desarrollada con posterioridad a los trabajos de Vygotsky (Vygostky, 1964) en la que se señalan distintos niveles de análisis de la actividad. La escuela socio-histórica estudia el desarrollo intelectual entendido a partir de un sustrato biológico y mediado por la cultura, donde el término cultural se refiere a todos los instrumentos, herramientas o productos de una cultura que han ido acumulándose a lo largo del tiempo como útiles para la funcionalidad del ser humano. La cultura así entendida proporciona las condiciones de posibilidad para actuar y, en último término, realizar actividades. 
Cuando las "herramientas culturales" convencionales no son suficientes o útiles para facilitar el desarrollo de una persona, es necesario hacer una determinada adaptación de dichos instrumentos. Vygotsky plantea que este proceso se lleva a cabo como una "mediación", es decir, se proporcionan los medios necesarios para poder conseguir el objetivo, medios que son culturales. En general, los instrumentos de mediación se pueden clasificar en dos tipos: materiales y simbólicos. Así, se entiende la actividad humana como producto de la interacción de, al menos, tres elementos: el sujeto, el entorno y la actividad o tarea en sí misma.

Según Leontiev (Leontiev, 1989) es posible diferenciar tres niveles en la actividad: actividad, acción y operaciones. La actividad es entendida como una concretización individual, que tiene un origen social y es útil para la satisfacción de necesidades. Rosa, Huertas y Blanco (Rosa, Huertas y Blanco, 1996) lo explican del siguiente modo:

"Ésta la entendemos aquí como la faceta individual de la práctica social. Mientras, podemos considerar que el grupo realiza prácticas sociales y el individuo se incorpora a éstas a través de la realización de actividades concretas (p.e.: trabajar, estudiar, ir a clase, escribir, etc.). Toda la actividad, por tanto, tiene un carácter social y está motivada, es decir, tiene un motivo concreto que la anima, aunque en ocasiones, este motivo puede no estar presente en la conciencia" (p. 330).
De este modo, la actividad es en su origen social y, por tanto está cargada culturalmente de valores, dirigida a un fin, teleológica y motivada para conseguir una determinada meta.

El segundo concepto, es la acción, que está en un nivel jerárquico inferior al de la actividad y superior al de las operaciones. La acción se diferencia de la actividad por dirigirse al logro de una meta concreta. Habitualmente una actividad puede incluir distintas acciones, del mismo modo que se pueden diferenciar distintas operaciones en una misma acción.

Rosa, Huertas y Blanco (op.cit.) ofrecen la siguiente definición: "La operación es lo que un sujeto concreto hace con unos instrumentos concretos en una situación concreta" (p. 332).

Las acciones toman lugar y se desarrollan en un contexto concreto, aspecto que, por un lado, nos permitirá la realización de actividades ofreciendo condiciones de posibilidad, y, por otro, nos limitará la ejecución o rendimiento. Estos conceptos y modelo suponen un mapa especular de las propuestas actuales sobre la discapacidad y autonomía (ver para su revisión la Clasificación Internacional del Funcionamiento, la Discapacidad y la Salud, 2001). Igualmente, estas ideas también se aproximan a las de Valsiner (Valsiner, 1997 , 2001) cuando utiliza el término "affordances".

En la Figura 1 se muestra cómo los tres términos se interrelacionan entre sí.

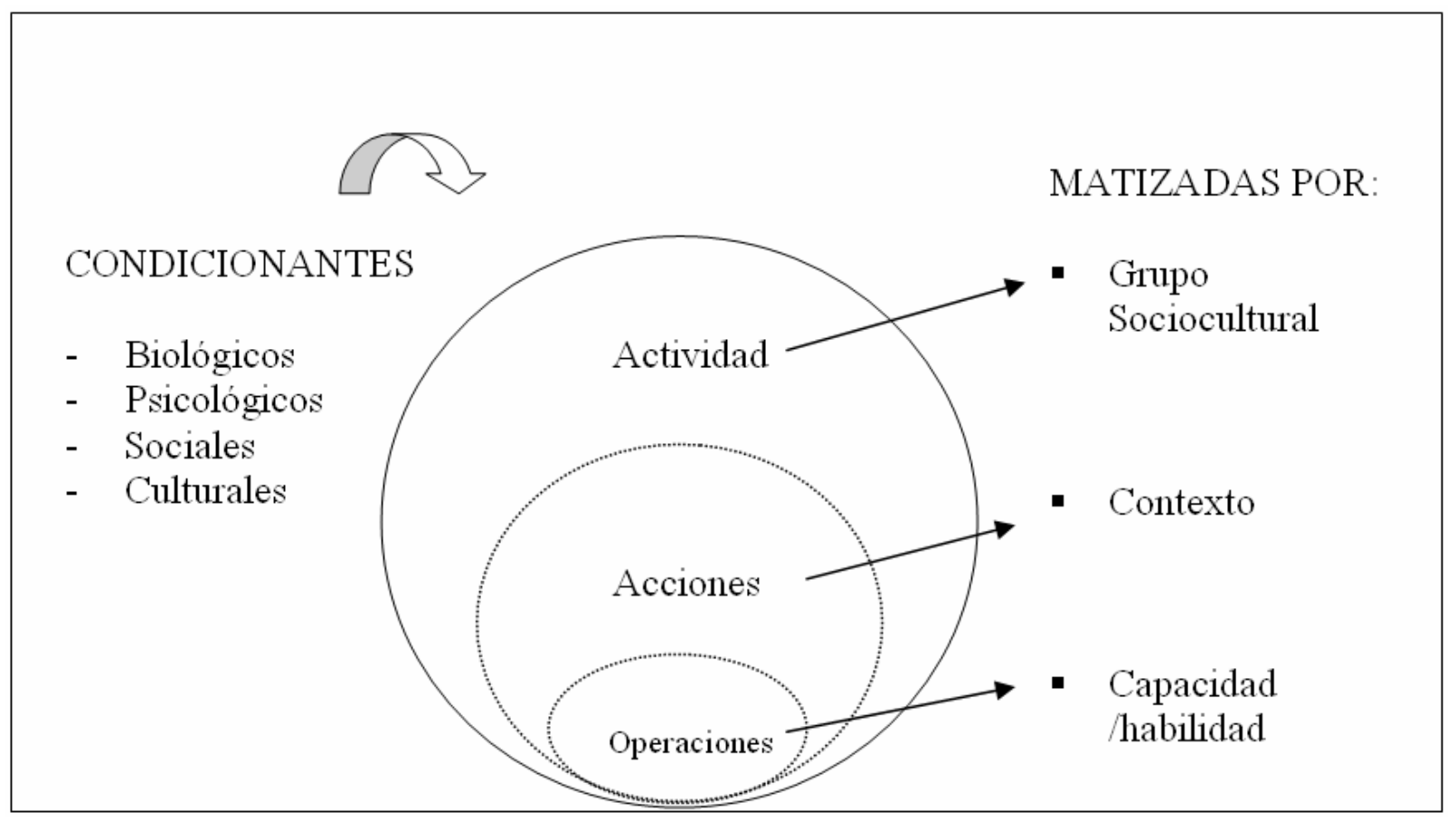

Figura 1: Teoría de Leontiev (Leontiev, 1989).

Otro autor de la misma tradición que Leontiev, Elkonin (Elkonin, 1971), hace una reflexión y propuesta sobre el desarrollo psicológico a partir de la actividad, y diferencia entre actividades orientadas hacia el significado de la activi- dad humana (aprendizaje de objetivos, motivos y normas del grupo) y actividades mediante las cuales el niño adquiere las formas socialmente desarrolladas de acción sobre los objetos y las normas sobre sus distintos usos. Tradicionalmente las 
actividades de la vida diaria han sido clasificadas en básicas, que obedecería a la acción con los objetos de Elkonin, y actividades de la vida diaria sociales, que presentan mayor similitud con las actividades de acción social del mismo autor.

Por otro lado, la epistemología genética entiende que una de las características del ser humano es su tendencia, habilidad para interactuar y transformar el mundo exterior, su medio y contextos, a través de su capacidad simbólica y de reflexión así como por su desempeño, ejecución y participación en diversas actividades (Piaget, 1947). También, para Piaget, el niño debe construir sus capacidades a través de las interacciones con el ambiente. Los conceptos suponen elementos perceptivos e información basada en la actividad, que son los que constituyen el núcleo a partir del cual el sujeto puede identificar un objeto y le proporcionan un mecanismo que facilita un funcionamiento adaptativo. González (1995) recoge que tal es la importancia de la representación de las acciones, en las que se incluyen tanto el movimiento como las propiedades que de él resultan, que Piaget supone que esos movimientos y conductas motoras se interiorizan de tal forma que las interpreta como si se tratara de un código de representación de las actividades de movimiento y causalidad. Hay dos procesos que se han utilizado para explicar el desarrollo y la adaptación: la asimilación y acomodación. A través de la asimilación el sujeto va interiorizando el entorno con el que interactúa, mientras que mediante la acomodación el sujeto va modificando su estructura cognitiva para adaptarse a las demandas del entorno. Cuando se ha alcanzado un determinado nivel de competencia, se pueden atender a nuevas demandas del entorno que supondrán un nuevo desafío y desequilibrio. Aspectos que están presentes en los principales modelos sobre la ocupación humana, donde no sólo la competencia real, sino también la percepción de competencia suponen un elemento clave para entender las actividades cotidianas (ver Kielhofner, 2002; 2006; Trombly y Radomski, 2002, 2007; Law, 1998).

Desde la psicología cognitiva existe una lectura particular de la actividad humana, donde al menos se pueden identificar tres enfoques diferentes para el estudio de la actividad.

Una primera aproximación está dedicada al estudio de los procesos psicológicos subyacentes a la actividad, conocidos como modelos de arriba a abajo (top down). Por otro lado, los modelos de abajo a arriba (bottom up) proponen que son los objetos, el contexto de ejecución el que activa distintos procesos cognitivos, como la percepción visual o auditiva, memoria semántica, memoria procedimental y el sistema motor lo que finalmente conducirá a una respuesta, que deseablemente permita el uso correcto de los objetos y contexto. Una tercera opción, es la representada por las arquitecturas cognitivas, como las propuestas por Newell (1990) y Anderson $(1983,1990)$.

En general, la psicología cognitiva pretende descubrir cómo las representaciones mentales del individuo determinan su comportamiento. Se concibe al hombre con una orientación pragmática, que quiere controlar la realidad a través del establecimiento de planes y logro de metas (De Vega, 1998). De este modo, se entiende que la mente recibe e interpreta las situaciones determinando así la actividad o acción futura, para ello es preciso que adquiera la información, la represente, la identifica, organice y dé significado, la almacene y la recupere o pierda, dé energía, actividad, mantenimiento y dirija el sistema conductual, resuelva problemas, tome decisiones, produzca una respuesta motora o mental y regule su propia actividad (Gadner, 1987).

De acuerdo con la primera perspectiva, Humphreys, Forde y Riddoch (Humphreys, Forde y Riddoch, 2001), han propuesto que la ejecución de actividades, que conllevan múltiples pasos, dependen de distintos procesos cognitivos. Así, son dos los elementos esenciales para la ejecución de actividades. En primer lugar, las representaciones almacenadas en la memoria, que son diferentes en función del grado de automaticidad de la tarea, como esquemas detallados para una tarea rutinaria y como esquemas generales para tareas no rutinarias, a los que habría que añadir el uso de estrategias y la solución de problemas para realizar una actividad de manera eficaz. $Y$ en segundo lugar, los procesos de recuperación de la información, considerando tanto la conducta motora como la secuencia temporal.

En relación con el anterior modelo está la propuesta de los esquemas mentales (Shallice, 1982). Resumidamente, propone que la conducta está mediatizada por esquemas mentales que interpretan los inputs externos y especifican una respuesta o acción, aunque los estímulos pueden activar distintos esquemas a la vez, lo que supone que existe un procesador para solucionar los conflictos, que entra en funcionamiento de manera automática y actúa en situaciones que son familiares o rutinarias, como ocurre en la mayoría de las actividades básicas de la vida diaria. Cuando la tarea a realizar es nueva o se producen modificaciones en la misma, es preciso emplear más recursos cognitivos, y así como la puesta en marcha del sistema de control atencional supervisor, que velará para que la ejecución de la actividad sea lo más precisa posible; por ejemplo, cuando se está aprendiendo a realizar una tarea nueva o surge un imprevisto en la misma (Kimber y Farah, 1993; Burgess y Hitch, 1992, 1999; Rumelhart y Norman, 1982).

Según estos autores existe una jerarquía de información supraordenada, que genera esquemas generales de acción o esquemas detallados. Los primeros permitirán la generalización, mientras que los segundos son exclusivos de ciertas actividades, y ambos pueden ser dependientes del contexto.

Como advierte González Marqués (1995), la conducta es construida por el organismo: la situación estimular se define, por tanto, no sólo a partir de las energías físicas de los estímulos sino también de sus propias predisposiciones y precondiciones; los datos de la situación y los datos provenientes del propio organismo en forma de estados internos motivacionales o hábitos adquiridos, son transformados y manipulados activamente hasta llegar al nivel de propositividad consciente; del mismo modo, el organismo o el sujeto tam- 
bién interviene al seleccionar y ejecutar la respuesta. La teoría cibernética (Weiner, 1948), supuso un hito fundamental en el desarrollo de la actual psicología cognitiva, incluyendo la actividad humana, considerándola dirigida a un objetivo, es decir, propositiva, mediada por la intencionalidad y la motivación del sujeto.

Una de las características rescatadas de la comprensión actual de las actividades de la vida diaria es precisamente el estar orientada a una meta o fin y estar influidas ampliamente por la motivación del individuo. Asimismo, el concepto de retroalimentación queda claramente patente en las actividades de la vida diaria donde es inexcusable la consideración del entorno, ya sea físico, social o cultural en el desempeño y participación en diferentes actividades. Evidentemente, para que esto sea posible el concepto del ser humano requiere necesariamente la consideración de que es un ser con representaciones mentales de información, sobre sus objetivos, el entorno, y con la capacidad de modificar su comportamiento para lograr su fines.

Otro elemento importante que influirá en la psicología cognitiva y en el entendimiento de las actividades es la diferencia entre competencia y actuación como herencia de la perspectiva chomskiana, que incluso ha quedado reflejado en el nuevo modelo de la Organización Mundial de la Salud en la clasificación del funcionamiento, discapacidad y participación (en adelante CIF).

Brenner, Ginsburg y Von Crananch advierten que aunque no existe un modelo único que represente el enfoque de la acción humana, se pueden identificar ciertos aspectos comunes a la mayor parte de los modelos de acción (Brenner, Ginsburg, \& Von Cranach, 1985). En primer lugar, entienden que la acción está organizada tanto jerárquica como secuencialmente. Enfatizan la importancia del contexto, incluyendo el contexto inmediato de las acciones antecedentes en la secuencia, el contexto situacional y la base sociocultural. Los procesos de feedback y feedforward, así como otros rasgos de los sistemas abiertos, son también comunes a estos modelos, como lo es una interacción explícita entre las categorías cognitiva y conductual de acción. Además, todos los modelos consideran que la acción está dirigida a la consecución de metas.

Por otro lado, la psicología de la acción representa un movimiento de integración entre la psicología cognitiva y conductual, rescatando la motivación y propositividad de la persona para realizar una determinada acción. Uno de los representantes de este enfoque es Atkinson que, junto a Birch, propuso la "Teoría Dinámica de la Acción" (Atkinson y Birch, 1978), siguiendo la línea de estudio planteada por Tolman (Tolman, 1925, 1932). En este sentido Garrido (Garrido, 1995) advierte que:

La acción se expresa en actividades, que se concretan y actualizan en actos. El concepto de actividad hace referencia a los mecanismos bio-psico-sociales a través de los que el sujeto manifiesta las acciones. La acción integra dos tipos de actividad, la actividad mental y la actividad física, que a su vez comprende distintos niveles (p. 481).
Según recoge Garrido (op.cit) la psicología de la acción está influida por la postura de Gibson (Gibson, 1960), en cuanto que la acción que la persona pueda realizar está posibilitada y a la vez limitada tanto por el entorno como por el propio individuo de una forma recíproca, lo que Gibson llama affordances del objeto y las effectivities del sujeto.

En íntima relación con la premisa anterior, es preciso considerar la característica o propiedad de poder realizar representaciones y llevar a cabo procesos de elaboración cognitiva que se verá influida tanto por la historia de aprendizaje y experiencia del individuo como por las circunstancias ambientales $\mathrm{o}$ condiciones de posibilidad.

En la psicología cognitiva ha predominado la tendencia de programas de investigación sobre aspectos parciales del procesamiento de la información. En este sentido, advierte Cañas que "se han propuesto modelos y realizado experimentos sobre sensación, percepción, memoria, etc. Muchas veces limitados a ciertas tareas especialmente diseñadas para realizar en el laboratorio y que poco tenían que ver con la vida real" (Cañas y Waerns, 2001, p.28 y 29).

Similarmente, Newell propuso crear teorías lo más generales posibles y que integrasen la mayor parte de las estructuras y procesos cognitivos, conocidas como arquitecturas cognitivas. Dentro de las arquitecturas cognitivas caben destacar dos originadas dentro de la ciencia cognitiva: ACT-R (Anderson, 1976) y SOAR (Newell, 1990) y otra propia de la investigación en ergonomía CCT (Kieras y Polson, 1985). ACT-R (Anderson, 1993) se originó a partir del modelo HAM de Anderson y Bower (1973) y posteriormente se amplió para explicar el aprendizaje y solución de problemas. Estas estructuras se apoyan en las reglas de producción (si "condición", entonces "acción") y en cómo el sistema cognitivo se adapta al ambiente. Se considera que el aprendizaje consiste en convertir el conocimiento declarativo en conocimiento procedimental, que se produce en tres etapas: 1) fase declarativa, donde el sujeto se repite mentalmente el conocimiento declarativo. Se utilizan las reglas de producción.; 2) fase de compilación; en función de si la regla ha tenido éxito en una tarea, se crea una regla nueva que es específica para un contexto donde se realiza la tarea. La compilación puede producirse de dos maneras: por composición, es decir, varias reglas se combinan en una sola regla; o por procedimentalización, cuando a una regla se le añade información específica de la tarea y del contexto; y 3) fase procedimental, cuando las reglas que han sido compiladas pueden ser automatizadas. Una vez que se ha producido el aprendizaje de una actividad, se puede producir una transferencia positiva a otra actividad, cuando una regla es aplicable, ó una transferencia negativa cuando la regla no es útil en otra situación.

En general, los diferentes marcos teóricos tienen en común, en primer lugar, que el ser humano es un ser activo, cuya actividad suele estar orientada hacia la consecución de un objetivo o meta, aspecto que a su vez conlleva la consideración de la intencionalidad, la elección, determinación, autonomía personal, la planificación de la acción, así como la 
resolución de los problemas previos, en el transcurso o terminación de la actividad. De este modo, la autonomía personal, la determinación y realización de una u otra actividad se contemplan no sólo como una necesidad, sino como una característica plenamente humana. Y en segundo lugar, entienden que toda acción tiene lugar en un determinado entorno y contexto que van a suponer un estímulo, desafío, un elemento facilitador o limitador.

\section{Las actividades de la vida diaria}

Dentro de las diferentes posibilidades de actuación hay un tipo de actividades que son comunes a las distintas culturas y tiempos y tienen que ver con la supervivencia y mantenimiento personal. Otras conductas son rutinarias, esperables $\mathrm{y}$, a veces, responden a las responsabilidades personales en función de los distintos roles. A estas actividades se las conoce habitualmente como actividades de la vida diaria (también conocidas como AVD).

El origen del término de las actividades de la vida diaria es relativamente reciente y surge dentro del ámbito de la salud. Su primer uso está relacionado con una lista de comprobación de treinta y siete actividades, en la obra titulada The physical demands of daily life (Deaver y Brown, 1945). No obstante, este médico y fisioterapeuta no llegaron a definir qué eran las actividades de la vida diaria. Cinco años más tarde, en 1950, se publicó otra escala de actividad de la vida diaria, esta vez desarrollada por terapeutas ocupacionales, dirigida a evaluar las destrezas de los niños con parálisis cerebral (Romero y Martorell, 2003).

Habrá que esperar a finales de los años 70 y primeros de los 80 para encontrar las primeras definiciones formales de las actividades de la vida diaria. La Asociación Americana de Terapia Ocupacional (A.O.T.A) elaboró la primera definición de actividades de la vida diaria (Moruno, 2003). En ella se indica que los componentes de las actividades diaria incluyen el cuidado personal, el trabajo y el juego o actividades lúdicas (Reed y Sanderson, 1999).

Más tarde, se definirían las actividades de la vida diaria como las tareas que una persona debe ser capaz de realizar para cuidar de sí mismo independientemente, incluyendo el cuidado personal, la comunicación y el desplazamiento (Reed y Sanderson, 1980).

Ann Catherine Trombly (Trombly, 1983) conceptualizaría del mismo modo las AVD, enfatizando el valor que tienen las mismas para el desempeño de los roles personales y profesionales. De manera muy similar, otros autores coinciden en considerar las actividades de la vida diaria como las tareas de mantenimiento personal, movilidad, comunicación, el manejo del hogar, que capacitan al individuo a lograr la independencia en su entorno (Pedretti, 1981; Mosey, 1986). Hay que observar que con el avance de la década de los años 80 se amplía el concepto, contemplando no sólo las actividades personales de independencia personal sino también aquellas que permiten tener independencia económica y autonomía en otros ámbitos cotidianos como las actividades de participación social, comunitaria y lúdica, aspecto que culminó en la década de 1990, con la escisión en actividades básicas e instrumentales de la vida diaria.

De este modo, se hace necesario diferenciar las AVD según el grado de complejidad cognitiva. Así, se puede hacer una taxonomía de las mismas en función de si se consideran básicas o instrumentales.

Las actividades básicas de la vida diaria (ABVD) se caracterizan por ser universales, estar ligadas a la supervivencia y condición humana, a las necesidades básicas, estar dirigidas a uno mismo y suponer un mínimo esfuerzo cognitivo, automatizándose su ejecución tempranamente (alrededor de los 6 años), con el fin de lograr la independencia personal. Habitualmente dentro de las ABVD se incluyen la alimentación, el aseo, baño, vestido, movilidad personal, sueño y descanso.

No obstante, conviene reconocer el hecho de que las actividades relacionadas con la supervivencia son actividades dependientes de cada cultura, que regula el modo para llevarlas a cabo adecuadamente, a través de rituales que en algunas ocasiones hacen difícil su asimilación y comprensión, así como su desempeño eficaz.

Poseen además, como dice Moruno (2003, p.15),

una función social básica, puesto que son indispensables para ser admitido y reconocido como un miembro perteneciente a una determinada comunidad. Es decir, constituyen el soporte mínimo para que se dé una integración social básica, permitiendo a cada sujeto realizar actividades que lo incorporan a lo social y, a la vez, se conforman en insignias que permiten reconocer a un individuo como perteneciente a una determinada cultura y sociedad.

En cambio, las actividades instrumentales de la vida diaria (AIVD) conllevan un mayor sesgo cultural, están ligadas al entorno, suelen ser instrumentales, frente a las primeras que son finalistas, son un medio para obtener o realizar otra acción, suponen una mayor complejidad cognitiva y motriz e implican la interacción con el medio, tales como utilizar distintos sistemas de comunicación, escribir, hablar por teléfono, movilidad comunitaria (conducir, uso de medios de transporte), mantenimiento de la propia salud, manejo de dinero, realización de compras, establecimiento y cuidado del hogar, cuidar de otro, uso de procedimientos de seguridad y respuesta ante emergencias.

No obstante la Clasificación Internacional de la Salud, la Discapacidad y el Funcionamiento (CIF, 2001), no hace la distinción de actividades básicas e instrumentales y proponen otra taxonomía:

- Aprendizaje y utilización del conocimiento: experiencias sensoriales intencionadas, aprendizaje básico, aplicación de conocimiento, resolución de problemas y toma de decisiones.

- Tareas y demandas generales: realización de tareas sencillas o complejas, organizar rutinas y manejar el estrés.

- Comunicación: recepción y producción de mensajes, llevar a cabo conversaciones y utilización de instrumentos y técnicas de comunicación. 
- Movilidad: cambiar y mantener la posición del cuerpo; llevar, mover y usar objetos; andar y moverse y desplazarse utilizando medios de transporte

- Autocuidado: lavarse y secarse, cuidado del propio cuerpo, vestirse, comer, beber y cuidar la propia salud.

- Vida doméstica: conseguir un lugar para vivir, comida, ropa y otras necesidades; tareas del hogar (limpiar y reparar el hogar, cuidar los objetos personales y de los del hogar y ayudar a otras personas.

- Interacciones y relaciones interpersonales: llevar a cabo interacciones interpersonales, particulares y generales de manera adecuada al contexto y entorno social.

- Áreas principales de la vida: educación, trabajo y empleo, y vida económica.

- Vida comunitaria, social y cívica: participación en la vida social fuera del ámbito familiar.

De cualquier modo, las actividades de la vida diaria están relacionadas con el logro de la independencia personal y la autonomía. En este sentido, se entiende que la independencia personal es la capacidad del individuo para satisfacer sus necesidades básicas, o realizar las actividades básicas de la vida diaria. En cambio, el término autonomía incluye además la independencia económica y la capacidad para tomar decisiones y obrar de acuerdo con las normas y creencias propias. De este modo, la situación de dependencia puede ser definida como el estado de carácter permanente en que se encuentran las personas que, por razones derivadas de la edad, la enfermedad o la discapacidad, y ligadas a la falta o a la pérdida de autonomía física, mental, intelectual o sensorial, precisan de la atención de otra u otras personas o ayuda importantes para realizar las actividades básicas de la vida diaria.

Las actividades de la vida diaria también construyen la identidad personal, están relacionadas con determinadas responsabilidades personales y sociales, son una forma de expresión y diferenciación personal.

En resumen, los rasgos distintivos que caracterizan a las actividades básicas e instrumentales de la vida diaria se pueden establecer en torno a:

- Los objetivos: es decir, las básicas son un tipo de actividad sobre la que se sustenta algo fundamental, esencial en oposición a la cualidad de mediadoras de las otras, aquellas de las que nos servimos para hacer algo, que utilizamos para lograr algo, y que pueden ser delegadas en otros.

- La complejidad: las básicas se definen por su sencillez o simplicidad en contraposición con la mayor complejidad de procesos cognitivos y sociales que define a las actividades instrumentales.

- La privacidad: Las actividades básicas, son personales frente a las instrumentales que pueden ser colectivas en el sentido de realizarse para interactuar con el entorno, públicamente sin necesidad de intimidad.

\section{¿Actividades de la Vida Diaria o Habilidades para la Vida Diaria?}

Se ha propuesto que sería mas correcto hablar de habilidades para la vida diaria en vez de actividades de la vida diaria. De acuerdo con las definiciones de distintos autores se entiende que las AVD pueden ser definidas como "la capacidad del individuo para llevar a cabo las actividades cotidianas esenciales" (Fernández-Ballesteros, 1992, p.80). En este sentido, podría entenderse como la capacidad o competencia del propio individuo para llevar a cabo las actividades de la vida diaria:

habría que utilizar la expresión habilidades para la vida diaria (HVD), más que la comúnmente utilizada actividades de la vida diaria (AVD) (Fernández-Ballesteros, 1992, p.151).

Es posible que en este punto de la lectura nos preguntemos ¿qué diferencia hay entre las habilidades adaptativas y las actividades de la vida diaria?, ¿cuál es la relación entre ambas? Lo cierto es que dependiendo del origen o campo de aplicación y de los autores se ha venido utilizando más una terminología que otra. Sin embargo, en general podemos afirmar que el término de habilidades adaptativas surge del ámbito del retraso mental, al estudiar cierto tipo de habilidades funcionales que son necesarias desarrollar para lograr la máxima independencia y autonomía personal (AARM, 2002 y Schadock, 1999). En cambio, el término de actividades de la vida diaria es más común en el ámbito de estudio de la discapacidad en general, como se expuso en el epígrafe anterior al hablar de su origen.

A continuación revisaremos brevemente qué se entiende por habilidades adaptativas, primero atendiendo a su etimología y posteriormente en el ámbito específico de la psicología.

Atendiendo a su origen etimológico, el término habilidad (Del lat. habilittas, -ātis) según el Diccionario de la Real Academia Española, significa "capacidad y disposición para algo, gracia y destreza en ejecutar algo que sirve de adorno a la persona, como bailar, montar a caballo, etc; Cada una de las cosas que una persona ejecuta con gracia y destreza". También, se podría entender como la competencia que alguien tiene para lograr un determinado objetivo. Mientras que, el término actividad (Del lat. activittas, $-\bar{a} t i s$ ) se refiere al "conjunto de operaciones o tareas propias de una persona o entidad, estando más relacionada con el desempeño o cumplimiento de las obligaciones inherentes a una profesión, cargo u oficio o dedicarse a una actividad" (DRAE, 2001).

Dentro de las habilidades adaptativas se han incluido principalmente las habilidades para el autocuidado personal, habilidades motrices, sociales, cognitivas, comunicativas y en algunos casos también profesionales. En la Tabla 1 mostramos un resumen de las distinciones hechas por los diferentes autores en los últimos años. 
Tabla 1: Clasificaciones de las habilidades adaptativas.

\begin{tabular}{|c|c|}
\hline Autor(es) & Dominios \\
\hline $\begin{array}{l}\text { Meyers, Nihira, y } \\
\text { Zetlin (1979) }\end{array}$ & $\begin{array}{l}\text { Habilidades de autoayuda } \\
\text { Desarrollo físico } \\
\text { Habilidades de comunicación } \\
\text { Funcionamiento cognitivo } \\
\text { Actividades domésticas y ocupacionales } \\
\text { Autodirección y responsabilidad } \\
\text { Socialización }\end{array}$ \\
\hline Kamphaus (1987). & $\begin{array}{l}\text { Físico/Motor } \\
\text { Autoayuda/Independencia } \\
\text { Interpersonal/Social } \\
\text { Responsabilidad } \\
\text { Cognitiva/Comunicación }\end{array}$ \\
\hline $\begin{array}{l}\text { McGrew y Bruininks } \\
\text { (1989) }\end{array}$ & $\begin{array}{l}\text { Independencia personal } \\
\text { Responsabilidad Personal } \\
\text { Responsabilidad Social } \\
\text { Habilidades Académicas Funciona- } \\
\text { les/Cognitivo } \\
\text { Profesional/Comunidad } \\
\text { Físico/Evolutivo }\end{array}$ \\
\hline $\begin{array}{l}\text { Widaman, } \quad \text { Borth- } \\
\text { wick-Duffy y Little } \\
\text { (1991) }\end{array}$ & $\begin{array}{l}\text { Desarrollo Motor } \\
\text { Habilidades para la Vida Independiente } \\
\text { Competencia Cognitiva } \\
\text { Competencia Social }\end{array}$ \\
\hline $\begin{array}{l}\text { Widaman, Stacy } \\
\text { y Brotwiuck-Duffy } \\
\text { (1993) }\end{array}$ & $\begin{array}{l}\text { Competencia Cognitiva } \\
\text { Competencia Social } \\
\text { Desadaptación Social } \\
\text { Desadaptación Personal }\end{array}$ \\
\hline Verdugo (1996) & $\begin{array}{l}\text { Comunicación } \\
\text { Autocuidado } \\
\text { Vida en el hogar } \\
\text { Habilidades sociales } \\
\text { Utilización de la comunidad } \\
\text { Autodirección } \\
\text { Salud y seguridad } \\
\text { Académicas funcionales } \\
\text { Ocio y tiempo libre } \\
\text { Trabajo }\end{array}$ \\
\hline
\end{tabular}

De este modo, y atendiendo a la revisión bibliográfica realizada parece que el término actividad se adecúa mejor al concepto aquí tratado: las actividades de la vida diaria. Así, se reconoce no sólo la importancia e interés que tienen las

\section{Referencias}

American Association of Mental Retardation (AAMR). (2002). Retraso Mental. Definición, clasificación y sistemas de apoyo. (10 ed.). Madrid: Alianza Editorial.

Anderson, J.R. y Bower, G.H. (1973). Human Associative Memory. Washington. Wisnston \& Sons.

Anderson, J.R. (1983). The architecture of cognition. Cambriedge, MA: Harvard University Press.

Anderson, J.R. (1990). The adaptive character of thought. Hillsdale. NJ: Erlbaum. Atkinson, J. y Birch, D. (1978). Introduction to motivation (2 $2^{\mathrm{a}}$ ed.). Nueva York: Van Nostrand. destrezas de una determinada persona sino, que su esencia es el producto de la interacción entre las destrezas del sujeto, las tareas u operaciones a realizar y el contexto donde se lleva a cabo, así como los roles que satisface con las actividades que realiza, que constituyen un elemento esencial en la formación y mantenimiento de la identidad personal.

\section{Conclusiones}

Las actividades de la vida diaria abarcan las actividades más frecuentes que realiza un sujeto, están relacionadas con lo familiar, diario, cotidiano, con las necesidades humanas, con la independencia y con el uso del tiempo. En función de su complejidad cognitiva y fin, hacia uno mismo o en relación con el entorno, se han establecido dos niveles: actividades básicas y actividades instrumentales de la vida diaria. En el origen de esta clasificación ya se alude a la importancia y necesidad de contemplar los procesos cognitivos subyacentes a la actividad. De este modo, las actividades de la vida diaria no pueden reducirse a la mera conducta motora observable.

Los primeros usos del término se hallan en el ámbito de la medicina de la rehabilitación y actualmente constituye unos de los pilares del ejercicio profesional de los terapeutas ocupacionales.

Aunque en algunos textos se emplea el término de habilidades de la vida diaria o habilidades adaptativas, éste hace más énfasis en la capacidad del individuo, mientras que el concepto y término de actividades de la vida diaria incluye también la consideración de las tareas concretas y su relación con el sujeto, con el contexto y entorno donde toman lugar y con su importancia en la construcción y mantenimiento de la identidad personal y satisfacción de los roles y obligaciones de cada individuo. Además, las actividades de la vida diaria están estructuradas temporal y secuencialmente. Su desempeño está relacionado con la percepción de competencia e identidad personal, los intereses, con el desarrollo de hábitos y estrategias.

Estos aspectos toman mayor trascendencia en el ámbito aplicado y el establecimiento de programas de rehabilitación, donde es imprescindible considerar la tarea, el contexto y al sujeto.

Brenner, M., Ginsburg, G. y Von Cranach, M. (1985). The human action approach: Summary of contributions. En G. P. Ginsburg, M. Brenner y M. Von Cranach (Eds.), Discovery strategies in the psychology of action. Londres: Academic Press.

Bruner, J. (1998). Actos de significado. Más allá de la revolución cognitiva. Madrid: Alianza editorial.

Burgess, N. y Hitch, G.J. (1992). Toward a newtwork model of the articulatory loop. Journal of Memory and Language, 31, 313-348. 
Burgess, N. y Hitch, G.J. (1999). Memory for serial order: A network model of the phonological loop and its timing. Psychological Review, 106, 551581.

Canter, D. (1985). Facet Theory: Approaches to social research. Nueva York: Spinger Verlag.

Cañas, J.J. y Waerns, Y. (2001). Ergonomía cognitiva: aspectos cognitivos de la interacción de las personas con la tecnología de la información. Madrid: Editorial Médica Panamericana.

Deaver, G.G. y Brown, M.E. (1945). Physical demands of daily life. New York: Institute for the crippled and disabled.

De Vega, M. (1998). Introducción a la psicología cognitiva. Madrid: Alianza Psicología.

Fernández-Ballesteros, R. I. (1992). Actividades de la vida diaria en la vejez. En R. I. Fernández-Ballesteros, Evaluación e intervención en la vejez: Barcelona: Martínez-Roca.

Gardner, H. (1987). La nueva ciencia de la mente. Historia de la revolución cognitiva. Buenos Aires: Paidós.

Garrido, I. (1995). Hacia la superación de sesgos y la integración en psicología: el modelo de la acción humana. Revista de Historia de la Psicología, 14 (3-4), 475-483.

Gibson, J. (1960). The concept of stimulus in psychology. American Psychologist, 15 (694-703).

González Marqués, J. (1995). El pensamiento. En J. Mora (Ed.), Psicología Básica III. Málaga: Edinfor.

Humphreys, G.W., Forde, E.M.E. y Riddoch, M.J. (2001). The planning and execution of everyday actions. En B. Rapp (Ed.), The handbook of cognitive neuropsychology. Philadelphia: Psychology Press.

Kielhofner, G. (2002). A model of human occupation: theory and application, $\left(3^{\mathrm{a}}\right.$ ed.). Baltimore: Lippincott, Williams \& Wilkins.

Kielhofner, G. (2006). Fundamentos conceptuales de la terapia ocupacional. Madrid: Editorial Médica Panamericana.

Kieras, D.E. y Polson, P.G. (1985). An approach to the formal analysis of user complexity. International Journal of Man-Machine Studies, 22, 163-182.

Kimberg, D.Y. y Farah, M.J. (1993). An unified account of cognitive impairments following frontal lobe damage: the role of working memory in complex, organized behaviour. Journal of Experimental Psychology: General, 122, 411-428.

Law, M. (Coord.) (1998). Client-centered occupational therapy. Thorofare, NJ: Slack.

Leontiev, A. (1989). The problem of activity in the history of Soviet Psychology. Soviet Psychology, 27(1), 22-39.

Mayor, J. (1985). Actividad humana y procesos cognitivos. Madrid: Alhambra.

Mayor, J. Suengas, A. \& González, J. (1993). Estrategias metacognitivas: aprender a aprender y aprender a pensar. Madrid: Síntesis

Moruno, P. (2006). Definición y clasificación de las actividades de la vida diaria. En P. Moruno y D. Romero (Eds.), Actividades de la vida diaria. Barcelona: Masson.
Mosey, A.C. (1996). Psychosocial components of occupational therapy. Philadelphia: Lippincott-Raven.

Neisser, U. (1967). Cognitive Psychology. Nueva York: Appleton.

Newell, A. (1990). Unified theories of cognition. Cambridge, Mass.: Harvard University Press.

OMS. (2001). Clasificación Internacional del Funcionamiento, la Discapacidad y la Salud (CIF). Ginebra: Organización Mundial de la Salud.

Pedretti, L.M. (1981). Occupational Therapy. Practice Skills for Physical Dysfunction. St. Louis: Mosby.

Piaget, J. (1947). La psicología de la inteligencia. Buenos Aires: Psique.

Real Academia Española (2001). Diccionario de la lengua español $\left(22^{\mathrm{a}}\right.$ ed.). Madrid: Espasa Calpe.

Reed, K. L. y Sanderson, S. (1980). Concepts of Occupational Therapy. Baltimore: Williams \& Wilkins.

Romero, D. y Martorell, M. (2003). Actividades básicas de la vida diaria o cuidado personal. En D. Romero y P. Moruno (Eds.), Terapia Ocupacional. Teoría y técnicas. Barcelona: Masson.

Rosa, A., Huertas, J. y Blanco, F. (1996). Metodología para la bistoria de la psicología. Madrid: Alianza.

Rumelhart, D.E. y Norman, D.A. (1982). Simulating a skilled typist: A study of skilled cognitive -motor performance. Cognitive Science, 6, 1-36

Schalock, R. L. (1999). Hacia una nueva concepción de la discapacidad. Siglo Cero, 30 (1), 5-20.

Shallice, T. (1982). Specific impairments of planning. Philosophical Transactions of the Royal Society of London, B 298, 199-209.

Tolman, E. (1925). Purpose and cognition: The determinants of animal learning. Psychological Review, 32, 285-297.

Tolman, E. (1932). Purpositive behavior in animals and men. Appleton: CenturyCrofts.

Trombly, C.A. y Radomski, M. (2002). Occupational therapy for physical dysfunction, ( $5^{\mathrm{a}}$ ed.). Philadelphia: Lippincott, Williams \& Wilkins.

Trombly, C.A. y Radomski, M. (2007). Occupational therapy for physical dysfunction, ( 6 a ed.). Philadelphia: Lippincott, Williams \& Wilkins.

Valsiner, J. (1997). Culture and the development of children's action. A theory of buman development. Nueva York: John Wiley \& Sons, Inc.

Valsiner, J. (2001). Process Structure of Semiotic Mediation in Human Development. Human Development, 44, 84-97.

Verdugo, M. (2006a). Autodeterminación y Calidad de Vida en Salud Mental: Dos conceptos emergentes. Salud Mental, 25(4), 68-77.

Verdugo, M. (2006b). Programa de habilidades de la vida diaria para la mejora de la conducta autodeterminada en personas con enfermedad mental grave y prolongada. Salamanca: Publicaciones del INICO. Universidad de Salamanca.

Vygostky, L. S. (1964). Pensamiento y lenguaje. Buenos Aires: La Pléyade. Weiner, N. (1948). Cybernetyic. Nueva York: Wiley.

(Articulo recibido: 16-4-2007; aceptado: 11-10-2007) 